\title{
Can magnifying endoscopy with narrow band imaging be useful for low grade adenomas in preoperative biopsy specimens?
}

\author{
Kazuhiro Miwa · Hisashi Doyama · Renma Ito - Hiroyoshi Nakanishi $\cdot$ Katsura Hirano $\cdot$ Satoko Inagaki \\ Kei Tominaga $\cdot$ Naohiro Yoshida $\cdot$ Kenichi Takemura $\cdot$ Shinya Yamada $\cdot$ Yoshibumi Kaneko $\cdot$ \\ Kazuyoshi Katayanagi $\cdot$ Hiroshi Kurumaya $\cdot$ Toshihide Okada $\cdot$ Masakazu Yamagishi
}

Received: 11 January 2011/Accepted: 28 August 2011/Published online: 13 March 2012

(c) The International Gastric Cancer Association and The Japanese Gastric Cancer Association 2012

\begin{abstract}
Background In biopsy specimens with low grade adenomas, it is often difficult to identify the presence of high grade adenomas or early carcinomas and low grade adenomas preoperatively, and clear guidelines have not yet been defined for the applicability of endoscopic treatment to low grade adenomas identified in biopsy specimens.

Methods We aimed to clarify the usefulness of magnifying endoscopy with narrow band imaging (NBI) compared to conventional white light endoscopy for diagnosing actual high grade adenomas or early carcinomas with low grade adenomas, using the VS (microvascular pattern $[\mathrm{V}]$ and microsurface pattern $[\mathrm{S}]$ ) classification for low grade adenomas in biopsy specimens. The study cohort consisted of 135 patients who were diagnosed with low grade adenomas in preoperative biopsy specimens and received endoscopic submucosal dissection.
\end{abstract}

K. Miwa $\cdot$ H. Doyama $\cdot$ R. Ito $\cdot$ H. Nakanishi $\cdot$ K. Hirano

S. Inagaki $\cdot$ K. Tominaga $\cdot$ N. Yoshida $\cdot$ K. Takemura $\cdot$

S. Yamada · Y. Kaneko

Department of Gastroenterology, Ishikawa Prefectural Central

Hospital, Kanazawa, Ishikawa, Japan

K. Miwa ( $\square)$

Department of Gastroenterology, Kanazawa Social Insurance

Hospital, Ha-15 Okimachi, Kanazawa, Ishikawa 920-8610,

Japan

e-mail: kzmiwatoureg@yahoo.co.jp

K. Katayanagi · H. Kurumaya

Department of Pathology, Ishikawa Prefectural Central Hospital,

Kanazawa, Ishikawa, Japan

T. Okada $\cdot$ M. Yamagishi

Division of Cardiovascular Medicine, Kanazawa University,

Kanazawa, Ishikawa, Japan
Results In the elevated type of lesion, magnifying endoscopy with NBI diagnosed high grade adenomas or early carcinomas at a higher sensitivity and specificity than conventional white light endoscopy (82.4 vs. $70.6 \%$, $P=0.391,97.3$ vs. $54.7 \%, P<0.0001)$. In the depressed macroscopic type of lesion, magnifying endoscopy with NBI also diagnosed high grade adenomas or early carcinomas at a higher sensitivity (95.5 vs. $68.2 \%, P=0.0459)$ than conventional white light endoscopy. Although the specificity was high, at $100 \%$, the difference when compared to conventional white light endoscopy was not significant (100 vs. $100 \%, P>0.99$ ).

Conclusions For low grade adenomas in biopsy specimens, it is vital to take sufficient consideration of endoscopic findings and not take action based only on the biopsy results. If a decision is made using the VS classification with magnifying endoscopy with NBI, actual high grade adenomas or early carcinomas can be differentiated from low grade adenomas so that endoscopic treatment can be performed more strictly.

Keywords Magnifying endoscopy $\cdot$ Narrow band Imaging · Low grade adenoma

\section{Introduction}

Gastric adenomas have been thought to rarely become malignant, as they are without findings of visual morphological change over long periods $[1,2]$. However, clinically, when lesions diagnosed as gastric adenomas in biopsy specimens were endoscopically removed, we often found that they were well-differentiated adenocarcinomas. The reasons for missing adenocarcinoma in biopsy material are that if the entire lesion is very well-differentiated 
adenocarcinoma, it is difficult to diagnose adenocarcinoma in a very small amount of biopsy material, or that the biopsy material is collected only from the section of the adenoma in a lesion that is adenocarcinoma in adenoma. Especially, it is recommended that high grade adenomas in biopsy specimens, diagnosed according to the Vienna classification of gastrointestinal epithelial neoplasia, should be removed, because it is highly probable that they will eventually be diagnosed as early carcinoma. But it is not clear how to handle low grade adenomas diagnosed in biopsy specimens. Thus, currently, there are divided opinions on whether low grade adenomas should be treated endoscopically in an aggressive way or whether they should be primarily observed, because many cases remain as adenomas for a long period [2, 3]. For low grade adenomas diagnosed in biopsy specimens, we think that it is important to actually identify cases of high grade adenomas or early carcinomas and cases of low grade adenomas endoscopically without being dependent upon the biopsy results. However, it is often difficult to identify these cases only by conventional white light endoscopy.

Recently, many new endoscopic devices have been developed, and as a result, a so-called optical biopsy has become possible. In particular, magnifying endoscopy was reported to be very useful for the diagnosis of superficial tumors throughout the entire gastrointestinal tract [4-6]. Of note is the development of a narrow band imaging (NBI) system, which is an electronic endoscope system using 2 wavelengths, of blue $(415 \mathrm{~nm})$ and green $(540 \mathrm{~nm})$, formed by spectral narrowing with consideration of the absorption

Table 1 Characteristics of patients

\begin{tabular}{|c|c|c|c|}
\hline & \multicolumn{2}{|c|}{$\begin{array}{l}\text { Diagnosis from ESD specimens } \\
\text { (Vienna classification) }\end{array}$} & \multirow[t]{2}{*}{$\begin{array}{l}\text { Total } \\
n=135\end{array}$} \\
\hline & $\begin{array}{l}\text { High grade } \\
\text { adenoma or early } \\
\text { carcinoma } \\
n=57\end{array}$ & $\begin{array}{l}\text { Low grade } \\
\text { adenoma } \\
n=78\end{array}$ & \\
\hline $\begin{array}{l}\text { Gender, male/ } \\
\text { female }\end{array}$ & $39 / 18$ & $38 / 40$ & $77 / 58$ \\
\hline $\begin{array}{l}\text { Age, years, median } \\
\text { (range) }\end{array}$ & $68.9(46-83)$ & $71.8(45-93)$ & $70.1(45-93)$ \\
\hline \multicolumn{4}{|l|}{ Macroscopic type } \\
\hline I & 6 & 6 & 12 \\
\hline IIa & 28 & 69 & 97 \\
\hline IIc & 23 & 3 & 26 \\
\hline \multicolumn{4}{|l|}{ Size (mm) } \\
\hline $3-9$ & 8 & 26 & 34 \\
\hline $10-19$ & 27 & 34 & 61 \\
\hline $20-29$ & 12 & 14 & 26 \\
\hline $30-$ & 10 & 4 & 14 \\
\hline
\end{tabular}

$E S D$ endoscopic submucosal dissection characteristics of hemoglobin. Microvascular patterns and microsurface patterns can be drawn clearly by using this NBI system along with magnifying endoscopy; thus, this modality has been used widely for the diagnosis of tumors in the gastrointestinal tract [7-9].

In Japan, a group classification has generally been used for the diagnosis of gastric biopsy materials. The group classification of gastric adenoma with mild and moderate atypia appears to correspond to low grade adenomas in the Vienna classification. In this study, we performed endoscopic submucosal dissection (ESD) for low grade adenomas diagnosed in biopsy specimens, and in the post-ESD diagnosis we retrospectively evaluated the differences in endoscopic findings using white light endoscopy and magnifying endoscopy with NBI for cases of low grade adenomas and cases of high grade adenomas or early carcinomas. Furthermore, we investigated the usefulness of magnifying endoscopy with NBI for the clinical handling of low grade adenomas diagnosed in biopsy specimens.

\section{Methods}

Subjects

We selected as subjects 135 consecutive patients (male: 77 , female: 58, mean age: 70.1 years) who were diagnosed with low grade adenoma in the preoperative biopsy specimen during the period from August 2006 through September 2009 and who received ESD. The macroscopic lesion type was elevated in 109 patients (type 0-I-like: 12 cases, type 0-IIa-like: 97 cases) and depressed in 26 patients (type 0-IIc-like) (Table 1).

\section{Endoscopy procedure}

As pretreatment for the endoscopic examination, in order to remove mucus on the gastric mucous membrane, 10,000 units of Pronase ${ }^{\circledR}$ (Kaken Pharmaceutical, Tokyo, Japan), $1 \mathrm{~g}$ of sodium bicarbonate, and $10 \mathrm{ml}$ of Balgin ${ }^{\circledR}$ (Kaigen, Tokyo, Japan) defoaming agent were dissolved in $100 \mathrm{ml}$ and subjects drank the solution $30 \mathrm{~min}$ prior to the examination. Endoscopy was performed by skilled endoscopy specialists. For magnification endoscopy with NBI and upper gastrointestinal tract magnifying endoscopy(GIF-Q240Z or GIF-H260Z; Olympus, Tokyo, Japan), an electronic endoscope (Evis Lucera Spectrum; Olympus) was used at our hospital and a video processor(CV260; Olympus) was set up as follows: color mode 1 and structure stressed B mode: level 4 during conventional white light endoscopy; and color mode 1 and structure stressed B mode: level 8 during magnifying endoscopy with NBI. In order to obtain stable endoscopic images at the maximal 


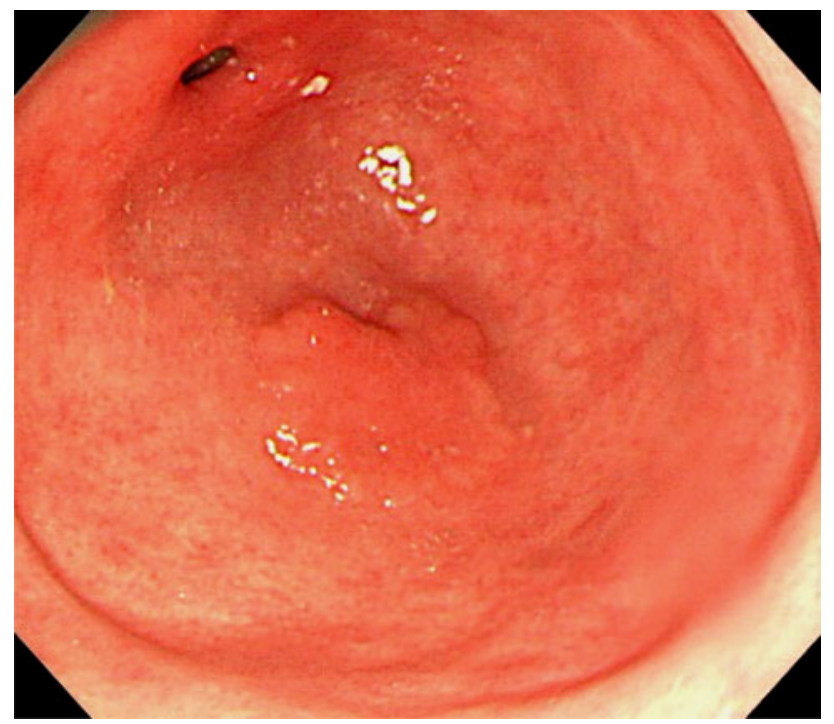

Fig. 1 Endoscopic findings of redness with conventional white light endoscopy

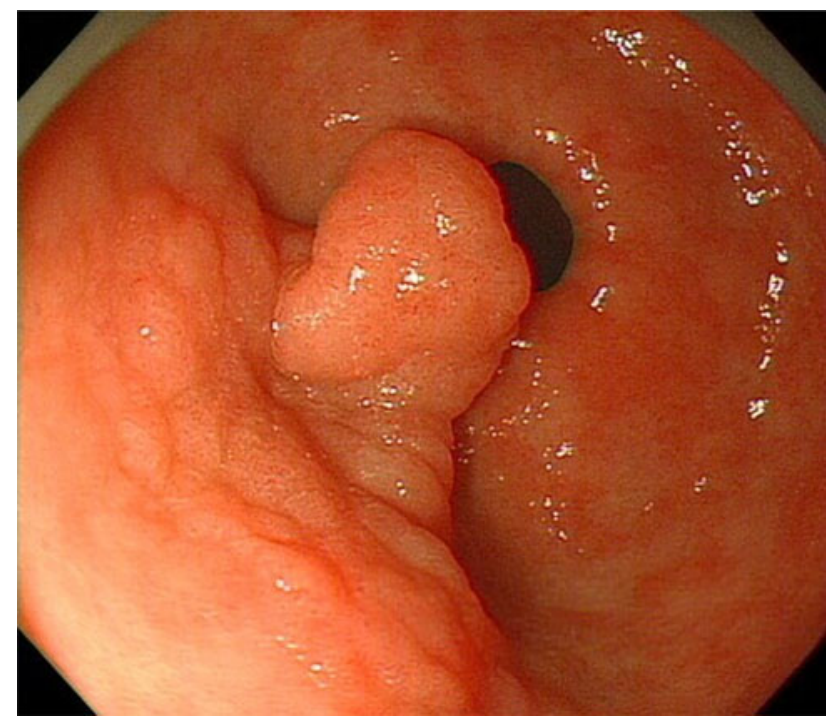

Fig. 2 Endoscopic findings of nodularity with conventional white light endoscopy

magnification, a black soft hood (MB-162 for GIF-Q240Z and MB-46 for GIF-H260Z; Olympus) was installed at the tip of the magnification endoscope.

Analysis of endoscopic findings

The endoscopic findings and recordings were reviewed in all cases and differences in the diameter of lesions and endoscopic findings between cases of low grade adenomas and cases of high grade adenomas or early carcinomas were investigated in the post-ESD diagnosis. The sizes of the lesions were measured after ESD, using a ruler. In the conventional white light endoscopy, we investigated the

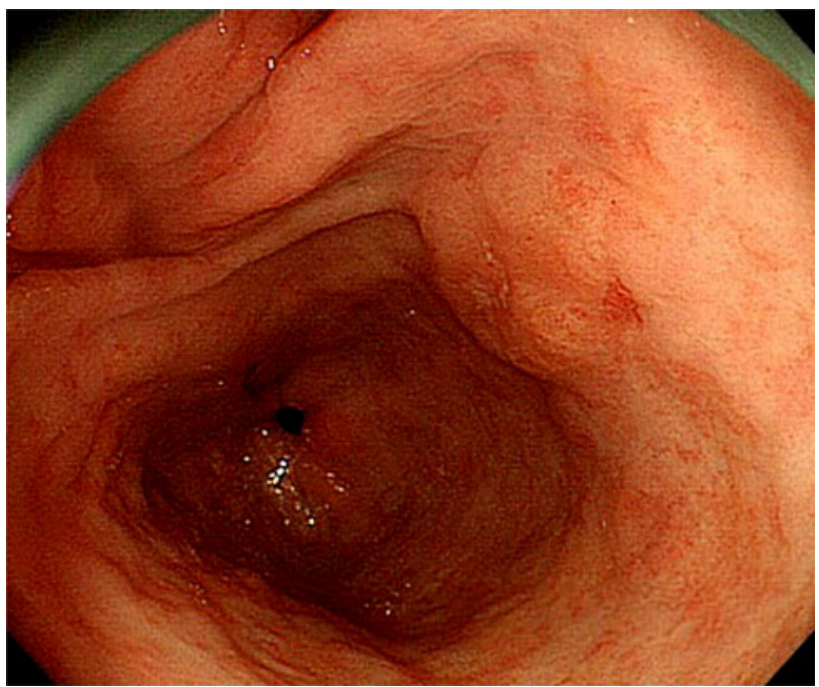

Fig. 3 Endoscopic findings of ulceration with conventional white light endoscopy

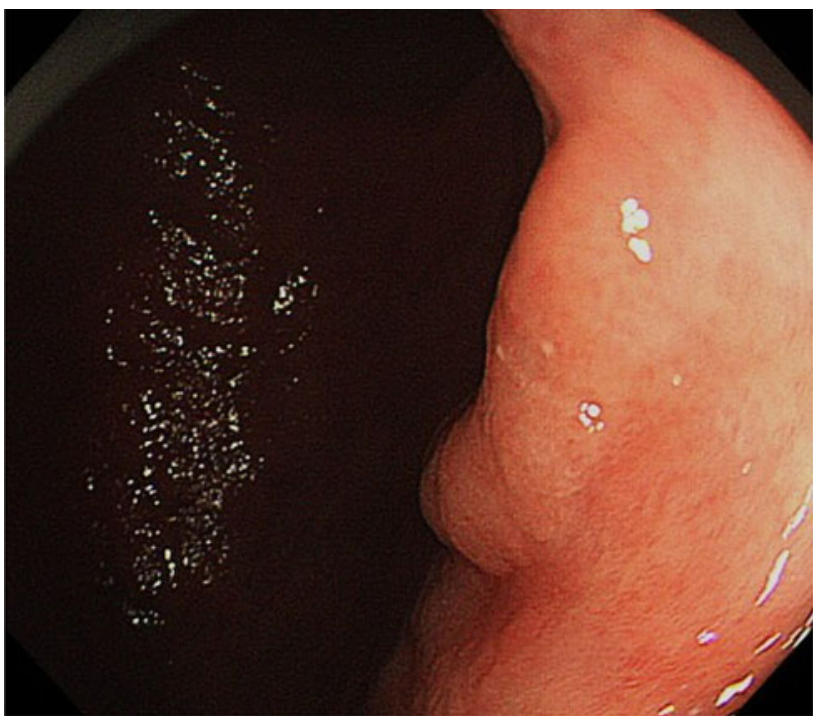

Fig. 4 Endoscopic findings of steep rise with conventional white light endoscopy

frequencies of lesions having the characteristic endoscopic findings reported as malignant transformation of gastric adenoma; that is, size more than $20 \mathrm{~mm}$ in diameter [10, 11], redness [12-14] (Fig. 1), nodularity [15, 16] (Fig. 2), ulceration [13] (Fig. 3), steep rise of edge [16] (Fig. 4), depression of surface appearance [13, 16] (Fig. 5) for elevated lesions, and irregular-shaped depression [15] (Fig. 6) for depressed lesions. In the magnifying endoscopy with NBI, the endoscopic findings were classified according to the definitions of the VS classification [17, 18] proposed by Yao et al. Namely, the microvascular pattern $(\mathrm{V})$ and microsurface pattern $(\mathrm{S})$ were each subclassified into 3 patterns: regular (Fig. 7), irregular (Fig. 8), and 


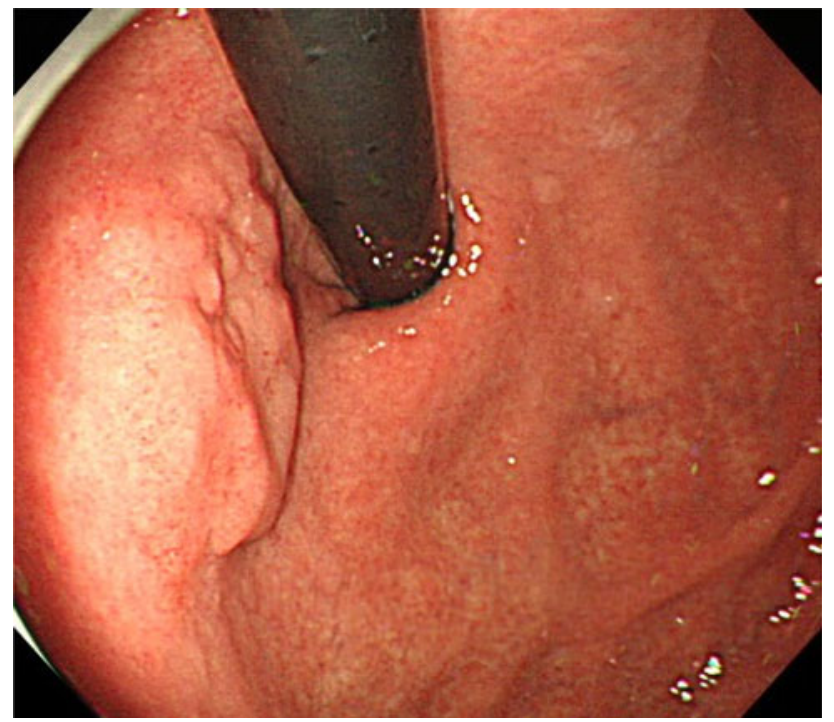

Fig. 5 Endoscopic findings of depressed elevated lesion with conventional white light endoscopy

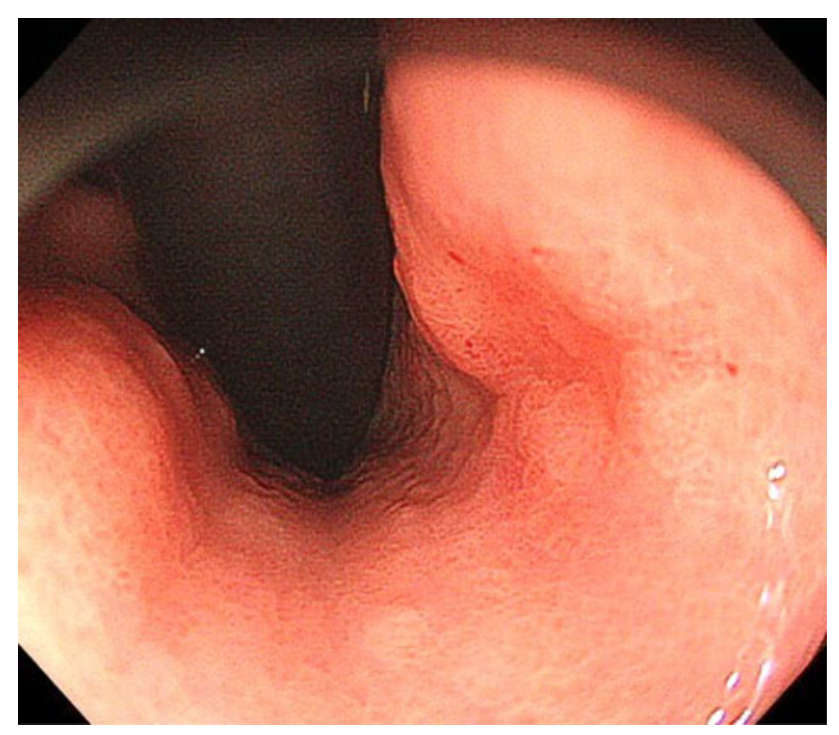

Fig. 6 Endoscopic findings of redness and irregular-shaped depression in depressed lesion with conventional white light endoscopy

absent (Figs. 9, 10), and the frequencies of each pattern were calculated.

\section{Pathological investigation}

All the endoscopic findings were evaluated retrospectively by 7 skilled endoscopy specialists, who reviewed both the white light and magnifying endoscopy images. Postoperative pathological diagnosis was performed by 2 pathologists and the results were double-checked for all cases.

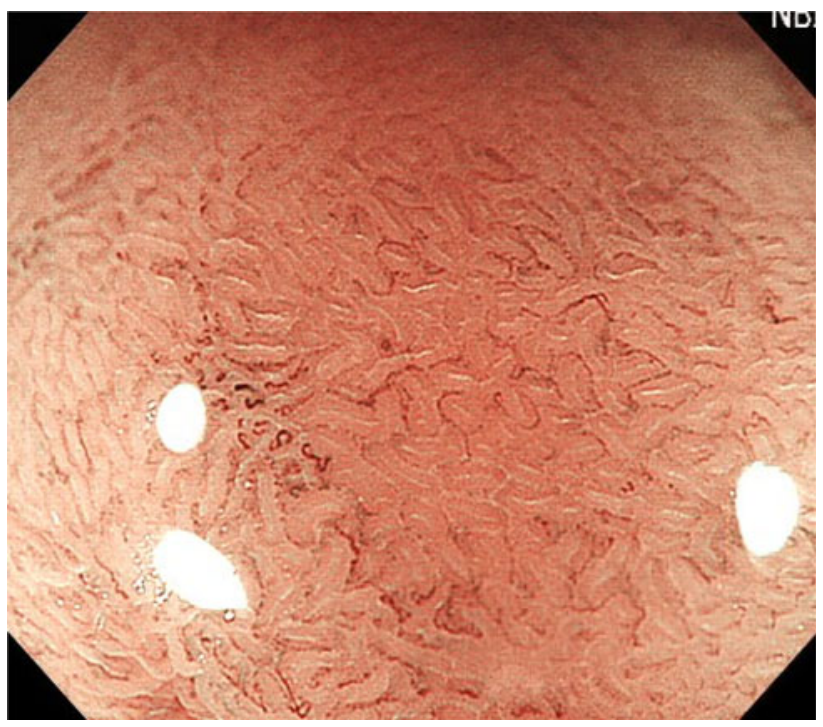

Fig. 7 Magnifying endoscopic findings with narrow band imaging (NBI) in elevated type of biopsy adenoma lesion. There is a regular microvascular pattern and a regular microsurface pattern

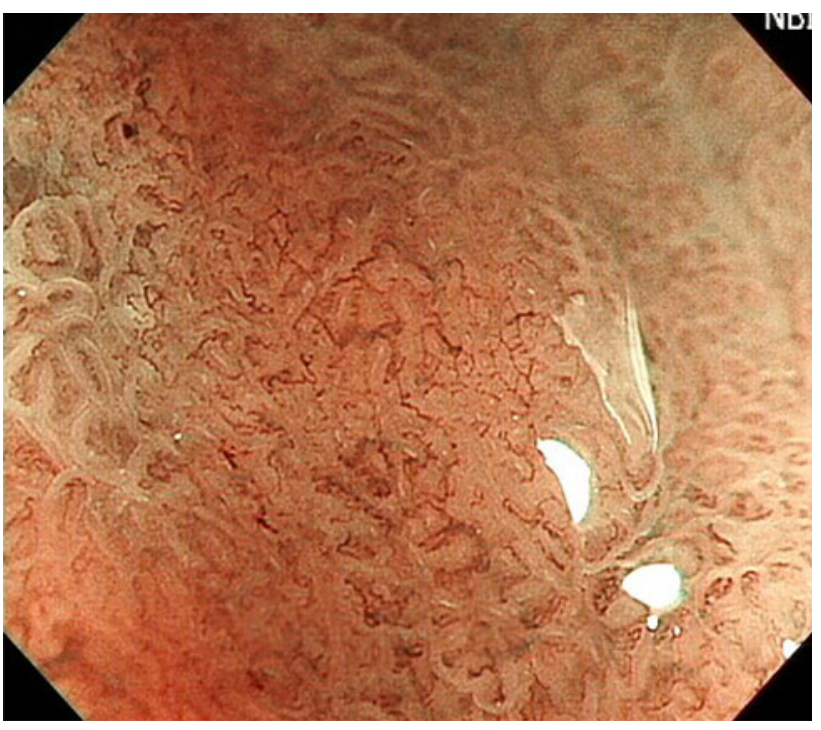

Fig. 8 Magnifying endoscopic findings with NBI in elevated type of biopsy adenoma lesion. There is an irregular microvascular pattern and an irregular microsurface pattern

\section{Statistics}

The Fisher direct probability method or the $\chi^{2}$ test were used for determining the frequencies of each endoscopic finding by conventional white light endoscopy and magnifying endoscopy with NBI. Regarding the distinctive conventional white light endoscopic findings for high grade adenomas or early carcinoma diagnosed from the ESD specimens, multiple logistic regression analysis was performed. All statistical indexes were determined to be 


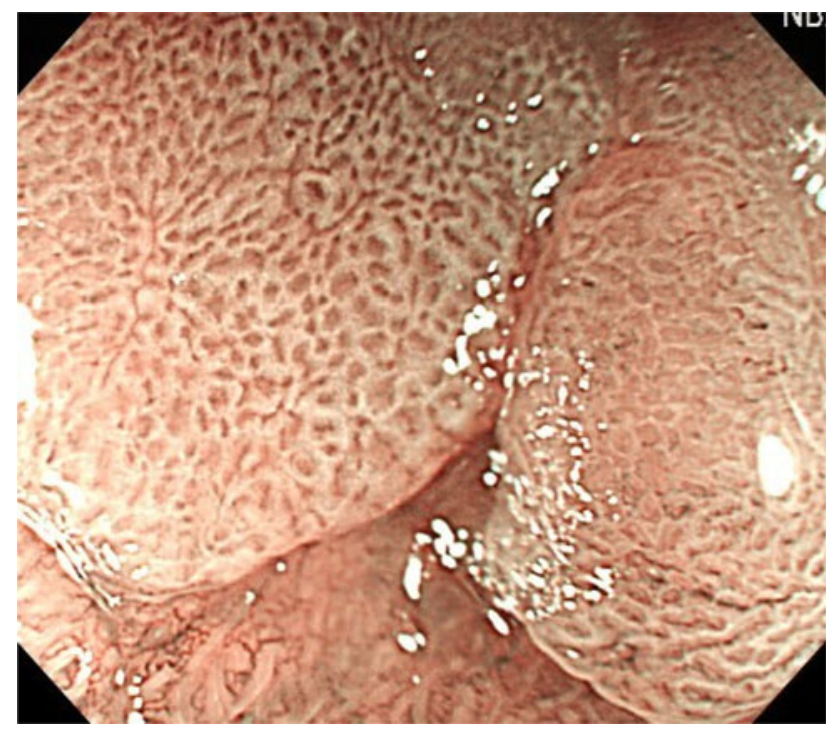

Fig. 9 Magnifying endoscopic findings with NBI in elevated type of biopsy adenoma lesion. The subepithelial microvascular pattern cannot be visualized because of a dense white opaque substance (WOS), so the microvascular pattern is absent. There is a regular microsurface pattern due to the regular distribution of WOS

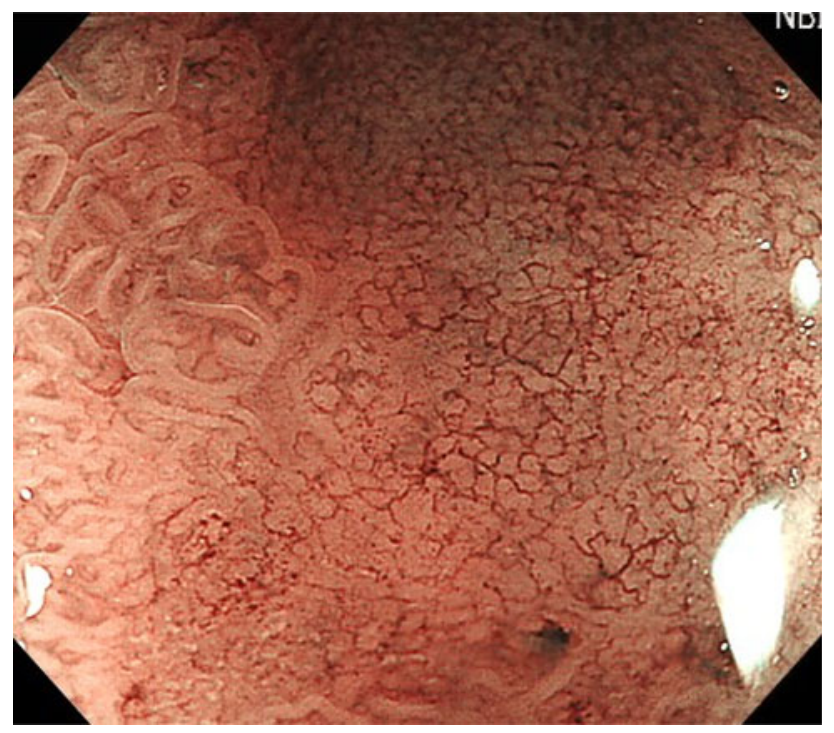

Fig. 10 Magnifying endoscopic findings with NBI in depressed type of biopsy adenoma lesion. The microvascular pattern is regular, and the microsurface architecture cannot be visualized, so the microsurface pattern is absent

significant with $P<0.05$ (Stat View 5.0 for Macintosh; SAS Institute Inc, Cary, North Carolina, USA).

\section{Results}

Fifty-seven of the 135 cases (42.2\%) were diagnosed as high grade adenomas or early carcinomas from the post-
Table 2 Comparison of findings of conventional white light endoscopy in 109 elevated lesions

\begin{tabular}{|c|c|c|c|}
\hline & \multicolumn{2}{|c|}{$\begin{array}{l}\text { Diagnosis from ESD specimens } \\
\text { (Vienna classification) }\end{array}$} & \multirow[t]{2}{*}{$P$ value } \\
\hline & $\begin{array}{l}\text { High grade adenoma } \\
\text { or early carcinoma } \\
n=34(\%)\end{array}$ & $\begin{array}{l}\text { Low grade } \\
\text { adenoma } \\
n=75(\%)\end{array}$ & \\
\hline Size $(\mathrm{mm})$ & & & 0.422 \\
\hline-19 & $18(53)$ & $56(75)$ & \\
\hline $20-$ & $16(47)$ & $19(25)$ & \\
\hline Redness & & & 0.035 \\
\hline$(+)$ & $18(53)$ & $17(23)$ & \\
\hline$(-)$ & $16(47)$ & $58(77)$ & \\
\hline Nodularity & & & 0.030 \\
\hline$(+)$ & $11(32)$ & $6(8)$ & \\
\hline$(-)$ & $23(68)$ & 69 (92) & \\
\hline Ulceration & & & $>0.999$ \\
\hline$(+)$ & $3(9)$ & 7 (9) & \\
\hline$(-)$ & 31 (91) & $68(91)$ & \\
\hline Steep rise & & & 0.0106 \\
\hline$(+)$ & $9(27)$ & $5(7)$ & \\
\hline$(-)$ & $25(73)$ & 70 (93) & \\
\hline Depression & & & 0.0024 \\
\hline$(+)$ & $12(35)$ & 7 (9) & \\
\hline$(-)$ & $22(65)$ & $68(91)$ & \\
\hline
\end{tabular}

ESD specimens. There were 3 cases of high grade adenomas $(5.3 \%)$ and 54 cases of early carcinomas $(94.7 \%)$. Seventy-eight cases were diagnosed as low grade adenomas $(57.8 \%)$.

Findings of conventional white light endoscopy

Table 2 shows comparisons of conventional white light endoscopy findings between the high grade adenomas or early carcinomas and the low grade adenomas diagnosed from ESD specimens in the 109 elevated type lesions. There were 34 cases diagnosed as high grade adenomas or early carcinomas from post-ESD specimens (31.2\%). As for the lesion diameter, when lesions were divided into a group of more than $20 \mathrm{~mm}$ and a group of less than $20 \mathrm{~mm}$, there was no significant difference between these two groups. As for the endoscopic findings, in the high grade adenomas or early carcinomas in the elevated lesions, the frequencies of redness $(P=0.035)$, nodularity $(P=0.03)$, steep rise $(P=0.011)$, and depression $(P=0.0024)$ were significantly higher when compared to the findings in the low grade adenomas However, there was no significant difference between these two groups in the frequency of ulceration. Table 3 shows the results of multivariate logistic regression analysis of these distinctive conventional white 
Table 3 Multivariate logistic regression analysis of the distinctive conventional white light endoscopic findings for high grade adenomas or early carcinomas diagnosed from ESD specimens

\begin{tabular}{lll}
\hline Factor & Odds ratio $(95 \%$ confidence interval $[\mathrm{CI}])$ & $P$ value \\
\hline Redness & $3.02(1.18-7.77)$ & 0.0217 \\
Nodularity & $3.68(1.03-13.2)$ & 0.0451 \\
Depression & $3.63(1.15-11.5)$ & 0.0283 \\
\hline
\end{tabular}

Table 4 Comparison of findings of conventional white light endoscopy in 26 depressed lesions

\begin{tabular}{ll} 
Diagnosis from ESD specimens & $P$ value \\
$\begin{array}{ll}\text { High grade adenoma or early } \\
\text { carcinoma } n=22(\%)\end{array}$ & $\begin{array}{l}\text { Low grade } \\
\text { adenoma } n=4 \\
(\%)\end{array}$ \\
\hline
\end{tabular}

\begin{tabular}{lll}
\hline Size $(\mathrm{mm})$ & & 0.5425 \\
$-19 \quad 16(73)$ & $4(100)$ & \\
$20-\quad 6(27)$ & $0(0)$ & 0.0987 \\
Redness & & \\
$(+) \quad 20(91)$ & $2(50)$ & \\
$(-) \quad 2(9)$ & $2(50)$ & \\
Nodularity & & \\
$(+) \quad 2(9)$ & $0(0)$ & 0.099 \\
$(-) \quad 20(91)$ & $4(100)$ & \\
Irregular-shaped depression & & \\
$(+) \quad 15(68)$ & $0(0)$ & \\
$(-) \quad 7(32)$ & $4(100)$ & \\
\hline
\end{tabular}

light endoscopic findings for high grade adenomas or early carcinoma in the elevated lesions diagnosed from the ESD specimens. From these results, the endoscopic findings of redness, nodularity, and depression were found to have a statistically significant relationship with deviation to malignant potential in the elevated lesions of low grade adenomas in biopsy specimens. Table 4 shows a comparison of conventional white light endoscopy findings between the high grade adenomas or early carcinomas and the low grade adenomas diagnosed from ESD specimens in the 26 depressed type lesions. Twenty-two lesions were diagnosed as high grade adenomas or early carcinomas from the postESD specimens (84.6\%). As for the lesion diameter, there was no significant difference between the groups with diameters of more than $20 \mathrm{~mm}$ and less than $20 \mathrm{~mm}$, as was found for the elevated type. No significant difference was found between the high grade adenomas or early carcinomas and the low grade adenomas in terms of endoscopic findings of redness, depression, and nodularity. The frequency of finding irregular-shaped depression was significantly higher $(P=0.0221)$ in the high grade adenomas or early carcinomas when compared to the low grade adenomas.
Table 5 Comparison of findings of magnifying endoscopy with narrow band imaging (NBI) in 109 elevated lesions

\begin{tabular}{llc}
\multicolumn{2}{l}{ Diagnosis from ESD specimens } & $P$ value \\
\cline { 1 - 2 } $\begin{array}{l}\text { High grade adenoma or } \\
\text { early carcinoma } n=34 \\
(\%)\end{array}$ & $\begin{array}{l}\text { Low grade } \\
\text { adenoma } \\
n=75(\%)\end{array}$ & \\
\hline ular pattern & $62(83)$ & $<0.0001$ \\
$3(9)$ & $12(16)$ & $>0.999$ \\
$5(15)$ & $1(1)$ & $<0.0001$ \\
$26(76)$ & & $<0.0001$ \\
pattern & $74(99)$ & 0.0083 \\
$5(15)$ & $0(0)$ & $<0.0001$ \\
$4(12)$ & $1(1)$ & \\
$25(73)$ & &
\end{tabular}

Findings of magnifying endoscopy with NBI

Table 5 shows a comparison of the findings of magnifying endoscopy with NBI between the high grade adenomas or early carcinomas and the low grade adenomas diagnosed from ESD specimens in the 109 elevated type lesions. Regarding the microvascular pattern, regular patterns were found in 62 cases $(83 \%)$ in the low grade adenomas, whereas regular patterns were found in only 4 cases $(10 \%)$ in the high grade adenomas or early carcinomas $(P<0.0001)$. Irregular patterns were found in 26 cases $(76 \%)$ in the high grade adenomas or early carcinomas, whereas irregular patterns were found in only 1 case $(1 \%)$ in the low grade adenomas $(P<0.0001)$.

As for the microsurface pattern, regular patterns were found in 74 cases (99\%) in the low grade adenomas, whereas regular patterns were found in only 5 cases $(15 \%)$ in the high grade adenomas or early carcinomas $(P<0.0001)$. Irregular patterns were found in 25 cases (73\%) in the high grade adenomas or early carcinomas, whereas irregular patterns were found in only 1 case $(1 \%)$ in the low grade adenomas $(P<0.0001)$.

Table 6 shows a comparison of the findings of magnifying endoscopy with NBI between the high grade adenomas or early carcinomas and the low grade adenomas diagnosed from ESD specimens in the 26 depressed type lesions. Regarding the microvascular patterns, regular patterns were found in 2 cases $(50 \%)$ in the low grade adenomas, whereas regular patterns were found in 3 cases $(14 \%)$ in the high grade adenomas or early carcinomas $(P=0.155)$. On the other hand, irregular patterns were found in 17 cases $(77 \%)$ in the high grade adenomas or early carcinomas, whereas none of the low grade adenomas showed irregular patterns $(P=0.0084)$. Regarding the microsurface patterns, regular patterns were found in 2 cases $(50 \%)$ in the low grade adenomas, whereas regular 
patterns were found in 2 cases $(9 \%)$ in the high grade adenomas or early carcinomas $(P=0.0987)$. Irregular patterns were found in 17 cases $(77 \%)$ in the high grade adenomas or early carcinomas, whereas none of the low grade adenomas had irregular patterns $(P=0.0084)$.

Comparison of the diagnostic accuracy for adenocarcinoma between conventional white light endoscopy and magnifying endoscopy with NBI

According to the results of the white light endoscopic findings diagnosed from the post-ESD specimens (Tables 2 and 3), we defined our diagnostic criterion using conventional white light endoscopy. That is, in the elevated type lesions, if at least one of the characteristic endoscopic findings of high grade adenoma or early carcinoma (namely, redness, nodularity, or depression) was found, we defined the lesions retrospectively as high grade adenomas or early carcinomas, and if there were no such findings, we defined the lesions as low grade adenomas. In the depressed type lesions, if there were endoscopic findings of irregular-shaped depression, we defined the lesions as high grade adenomas or early carcinomas, and if there were no findings of irregular-shaped depression, we defined the lesions as low grade adenomas.

Table 6 Comparison of findings of magnifying endoscopy with NBI in 26 depressed lesions

\begin{tabular}{|c|c|c|}
\hline Diagnosis from ESD spe & ens & $P$ value \\
\hline $\begin{array}{l}\text { High grade adenoma or } \\
\text { early carcinoma } n=22 \\
(\%)\end{array}$ & $\begin{array}{l}\text { Low grade } \\
\text { adenoma } n=4 \\
(\%)\end{array}$ & \\
\hline
\end{tabular}

Microvascular pattern

\begin{tabular}{ccll} 
Regular & $3(14)$ & $2(50)$ & 0.155 \\
Absent & $2(9)$ & $2(50)$ & 0.0987 \\
Irregular & $17(77)$ & $0(0)$ & 0.0084 \\
Microsurface & pattern & & \\
Regular & $2(9)$ & $2(50)$ & 0.0987 \\
Absent & $3(14)$ & $2(50)$ & 0.155 \\
Irregular & $17(77)$ & $0(0)$ & 0.0084 \\
\hline
\end{tabular}

According to the diagnostic criteria $[17,18]$ for early gastric cancer based on the VS classification; namely: (1) if the microvascular pattern is irregular and a demarcation line is detectable or (2) if the microsurface pattern is irregular and a demarcation line is detectable, the case is defined as high grade adenoma or early carcinoma when at least one of the findings in (1) or (2) is present, and the case is defined as low grade adenoma when these findings are absent; this applies to both elevated type and depressed type lesions shown on magnifying endoscopy with NBI.

In accordance with these diagnostic criteria, Table 7 shows the calculated sensitivity and specificity of magnifying endoscopy with NBI and conventional white light endoscopy for the diagnosis of high grade adenomas or early carcinomas. In the elevated type lesions, magnifying endoscopy with NBI was found to be capable of diagnosing high grade adenomas or early carcinomas at a higher sensitivity than conventional white light endoscopy (82.4 vs. $70.6 \%, P=0.391$ ), but the difference was not significant. Specificity compared to that of conventional white light endoscopy was significantly higher (97.3 vs. 54.7\%, $P<0.0001)$. In the depressed type lesions, magnifying endoscopy with NBI was found to be capable of diagnosing high grade adenomas or early carcinomas at a higher sensitivity compared to that of conventional white light endoscopy (95.5 vs. $68.2 \%, P=0.0459$ ). Specificity for both magnifying endoscopy with NBI and conventional white light endoscopy was high, at $100 \%$, although there was a small number of cases.

\section{Discussion}

Regarding the clinical handling of adenoma diagnosed in biopsy specimens, the rate of malignant transformation of adenomas increases in proportion to their increase in size, and adenomas larger than $2 \mathrm{~cm}$ in diameter are regarded as having malignant potential $[10,11]$. Park et al. [12] found that, in their univariate analysis, location, histological type, surface redness, and degree of dysplasia had a statistically significant relationship with malignant transformation. Jung et al. [13] also suggested that gastric adenomas with characteristics such as a depressed type, red color, and

Table 7 Comparison of diagnostic accuracy for adenocarcinoma between conventional white light endoscopy and magnifying endoscopy with NBI

\begin{tabular}{|c|c|c|c|c|c|c|}
\hline & \multicolumn{3}{|l|}{ Elevated lesions } & \multicolumn{3}{|l|}{ Depressed lesions } \\
\hline & $\begin{array}{l}\text { Conventional white light } \\
\text { endoscopy }(\%)\end{array}$ & $\begin{array}{l}\text { Magnifying endoscopy } \\
\text { with NBI (\%) }\end{array}$ & $P$ value & $\begin{array}{l}\text { Conventional white light } \\
\text { endoscopy }(\%)\end{array}$ & $\begin{array}{l}\text { Magnifying endoscopy } \\
\text { with NBI }(\%)\end{array}$ & $P$ value \\
\hline Sensitivity & 70.6 & 82.4 & 0.391 & 68.2 & 95.5 & 0.0459 \\
\hline Specificity & 54.7 & 97.3 & $<0.0001$ & 100 & 100 & $>0.999$ \\
\hline
\end{tabular}


ulceration, may have foci of carcinomas. Nakamura et al. [15] reported that depressed gastric adenomas had shallow depressions of a light-brown color, a round or oval shape, and irregular margins. On the other hand, Katsube et al. [19], in a report based on endoscopic mucosal resection (EMR) performed because of adenoma in biopsy specimens, noted that $37.2 \%$ of the patients' specimens showed adenocarcinoma and $62.8 \%$ showed adenoma and that there were no significant differences between the two groups of patients in terms of gender, age, sites of lesions, endoscopic findings, or lesion diameter.

However, the problem with the malignant potential of adenoma in biopsy specimens is that there are different opinions among reports as to the characteristic white light endoscopic findings. Recently, there have been several reports on the identification of adenocarcinomas and adenoma using magnifying endoscopy with NBI. Nakayoshi et al. [20] reported that most of the "small round", "round or oval", and "tubular" patterns showing relatively regular microsurface patterns were histologically adenomas, while "irregular" patterns and the appearance of abnormal vascular images showing disturbances in the microsurface pattern were more likely adenocarcinoma.

Regarding the identification and diagnosis of adenocarcinoma by magnifying endoscopy with NBI, Yao et al. [21] reported that, in many blinded prospective studies, the negative predictive value of the demarcation line was $100 \%$ and the presence of an irregular microvascular pattern indicated a diagnostic accuracy of $98.7 \%$. With respect to the ability to diagnose cancer when using the VS classification, Yao et al. [18] reported, in their study of 100 patients with early gastric cancer, that adenocarcinomas were detectable with a sensitivity of $97 \%$ and that all three cases that did not fulfill the VS diagnostic criteria had poorly differentiated adenocarcinoma. It was stated that this was the limitation of magnifying endoscopic diagnosis.

In the present study, all the cases of early carcinomas diagnosed from the post-ESD specimens were differentiated adenocarcinomas, and these results showed a slightly lower sensitivity compared with those reported by Yao et al. [18]. The reason for this lower sensitivity is assumed to be that these cases of low grade adenomas on biopsy specimens when compared to already diagnosed high grade adenomas or early carcinomas on biopsy specimens might have slight irregularities of microvascular patterns and microsurface patterns. In addition, we should pay attention to differences between pathologists in the diagnosis of adenoma and early carcinoma.

In elevated lesions in biopsy specimens, endoscopic findings of redness, nodularity, and depression in the conventional white light endoscopy for low grade adenomas are considered to be useful for diagnosing high grade adenomas or early carcinomas (Table 3 ). However, the sensitivity and specificity of conventional white light endoscopy for the diagnosis of high grade adenomas or early carcinomas were low (Table 7). In contrast, magnifying endoscopy with NBI was shown to be very useful for differentiating high grade adenomas or early carcinomas from low grade adenomas, due to its relatively higher sensitivity and specificity when compared with conventional white light endoscopy (Table 7). Depressed adenomas are relatively rare and their malignant potential is generally considered to be higher than that of the elevated type [22-24]. In the present study, the frequency of the depressed type of low grade adenomas was only $19.3 \%$ of the overall when diagnosed from the post-ESD specimens, but the frequency when diagnosed in biopsy specimens with high grade adenomas or early carcinomas was found to be significantly higher than that of the elevated type (84.6 vs. $31.2 \%, P<0.001$ ).

Although the finding of an irregular-shaped depression on conventional white light endoscopy was considered as a characteristic finding for diagnosing high grade adenoma or early carcinoma, the sensitivity of the diagnosis of high grade adenomas or early carcinomas when using magnifying endoscopy with NBI was significantly higher than that of the conventional white light endoscopy.

According to the forementioned diagnostic criteria for diagnosing high grade adenomas or early carcinomas of 7 cases that could not be diagnosed when using magnifying endoscopy with NBI, there were 4 cases $(57.1 \%$ ) (3 cases in elevated lesions, 1 case in a depressed lesion) that could be diagnosed using conventional white light endoscopy only. On the other hand, in 17 cases that could not be diagnosed when using conventional white light endoscopy, there were 15 cases $(88.2 \%$ ) (8 cases in elevated lesions, 7 cases in depressed lesions) that could be diagnosed using magnifying endoscopy with NBI only.

Generally, in patients with lesions of low grade adenoma in biopsy specimens there is a tendency of physicians to keep an eye on developments. But the problem is that some proportions of these lesions actually include high grade adenomas or early carcinomas. In the present study, 57 cases $(42.2 \%)$ overall were eventually diagnosed as high grade adenomas or early carcinomas. As to the handling of low grade adenomas in biopsy specimens, if the endoscopic findings using both conventional white light endoscopy and magnifying endoscopy with NBI do not satisfy the abovementioned criteria, the lesions can actually be regarded as low grade adenomas as shown in the biopsy specimens, so it should be clinically feasible to consider an endoscopic treatment when there are changes in the endoscopic findings after strict follow-up observation. On the other hand, if the endoscopic findings of magnifying endoscopy with NBI satisfy the diagnostic criteria of the VS classification, there is actually a very high possibility that the lesion is a high 
grade adenoma or early carcinoma even though the findings of conventional white light endoscopy have suggested a low grade adenoma, and therefore early endoscopic treatment should be considered.

In the future, further accumulation of cases and prospective studies are desirable. In conclusion, for low grade adenomas in biopsy specimens, it seems to be very important to take action with sufficient consideration of the endoscopic findings and not take action based only on the biopsy results. For this reason, if a decision is made using the VS classification with magnifying endoscopy with NBI, actual high grade adenomas or early carcinomas can be differentiated from low grade adenomas, so that endoscopic treatment can be performed more strictly.

\section{References}

1. Nagatomi Y, Kawamura S, Kawashima M, et al. Clinical followup of protruded atypical epithelial foci of the stomach. Gastroenterol Endosc. 1980;22:275-83.

2. Yamada H, Ikegami M, Shimoda T, Takagi N, Maruyama M. Long-term follow-up study of gastric adenoma/dysplasia. Endoscopy. 2004;36:390-6.

3. Hasuike N, Oda I, Gotoda T, Hamanaka H, Yamaguchi H, Kouzu $\mathrm{T}$, et al. Clinical management of gastric adenoma, from the point of view of endoscopic surveillance. Stomach Intest. 2003;10: 1419-26.

4. Inoue H, Kumagai Y, Yoshida T. High-magnification endoscopic diagnosis of superficial esophageal cancer. Dig Endosc. 2000;12: 532-5.

5. Yao K, Oishi T, Matsui T, Yao T, Iwashita A. Novel magnified endoscopic findings of microvascular architecture in intramucosal gastric cancer. Gastrointest Endosc. 2002;56:279-84.

6. Kudo S, Tamura S, Nakajima T, Yamano H, Kusaka H, Watanabe H. Diagnosis of colorectal tumorous lesions by magnifying endoscopy. Gastrointest Endosc. 1996;44:8-14.

7. Yoshida T, Inoue H, Usui S, Satodate H, Fukami N, Kudo SE. Narrow-band imaging system with magnifying endoscopy for superficial esophageal lesions. Gastrointest Endosc. 2004;59: 288-95.

8. Nakayoshi T, Tajiri H, Matsuda K, Kaise M, Ikegami M, Sasaki H. Magnifying endoscopy combined with narrow band imaging system for early gastric cancer: correlation of vascular pattern with histopathology (including video). Endoscopy. 2004;36: $1080-4$.

9. Hirata M, Tanaka S, Oka S, Kaneko I, Yoshida S, Yoshihara M, Chayama K. Magnifying endoscopy with narrow band imaging for diagnosis of colorectal tumors. Gastrointest Endosc. 2007:65:988-95.

10. Hirota T, Okada T, Itabashi M, Kitaoka H. Histogenesis of human gastric cancer: with special reference to the significance of adenoma as a precancerous lesion. In: Ming SC, editor. Precursors of gastric cancer. New York: Praeger; 1984. p. 232-52.

11. Tomasulo J. Gastric polyps: histologic types and their relationship to gastric carcinoma. Cancer. 1971;27:1346-55.

12. Park DI, Rhee PL, Kim JE, Hyun JG, Kim YH, Son HJ, et al. Risk factors suggesting malignant transformation of gastric adenoma: univariate and multivariate analysis. Endoscopy. 2001;33: $501-6$.

13. Jung MK, Jeon SW, Park SY, Cho CM, Tak WY, Kweon YO, et al. Endoscopic characteristics of gastric adenomas suggesting carcinomatous transformation. Surg Endosc. 2008;22:2705-11.

14. Kitoh T, Yanai H, Matsubara Y, Nakamura Y, Okamoto T, Hirano A, et al. Endoscopic findings potentially predictive of gastric cancer in borderline lesions diagnosed by forceps biopsy. Hepatogastroenterology. 2005;52:404-8.

15. Nakamura K, Sakaguchi H, Enjoji M. Depressed adenoma of the stomach. Cancer. 1988;15:2197-202.

16. Nakahara K, Watanabe Y, Tamiya Y, Serikawa O, Kominato K, Matsuo K, et al. Macroscopic type of early gastric cancer, type 0 I and type 0 IIa cancer. Stomach Intest. 2009;44:507-21.

17. Yao K, Takaki Y, Matsui T, Iwashita A, Anagnostopoulos GK, Kaye $\mathrm{P}$, et al. Clinical application of magnification endoscopy and narrow-band imaging in the upper gastrointestinal tract: new imaging techniques for detecting and characterizing gastrointestinal neoplasia. Gastrointest Endosc Clin N Am. 2008;18:415-33.

18. Yao K, Anagnostopoulos K, Ragunath K. Magnifying endoscopy for diagnosing and delineating early gastric cancer. Endoscopy. 2009;41:462-7.

19. Katsube T, Konno S, Hamaguchi K, Shimakawa T, Naritaka Y, Ogawa $\mathrm{K}$, et al. The efficacy of endoscopic mucosal resection in the diagnosis and treatment of group III gastric lesions. Anticancer Res. 2005;25:3513-6.

20. Nakayoshi T, Tajiri H, Saito S, Kaise M, Ikegami M, Matsuda K, et al. Magnifying endoscopy of gastric adenoma. Stomach Intest. 2003;38:1401-9.

21. Yao K, Iwashita A, Tanabe H, Nagahama T, Matsui T, Ueki T, et al. Novel zoom endoscopy technique for diagnosis of small flat gastric cancer, a prospective, blind study. Clin Gastroenterol Hepatol. 2007;5:869-78.

22. Xuan ZX, Ambe K, Enjoji M. Depressed adenoma of the stomach, revised. Histologic, histochemical, and immunohistochemical profiles. Cancer. 1991;1:2382-9.

23. Tamai N, Kaise M, Nakayoshi T, Katoh M, Sumiyama K, Gohda $\mathrm{K}$, et al. Clinical and endoscopic characterization of depressed gastric adenoma. Endoscopy. 2006;38:391-4.

24. Nakamura K, Sakaguchi H, Enjoji M. Depressed adenoma of the stomach. Cancer. 1988;15:2197-202. 